Artículos

\title{
La grieta en medios tradicionales y digitales
}

\section{A rachadura na mídia tradicional e digital The "Crack" in Traditional and Digital Media}

\author{
Demirdjian, Liliana
}

\author{
Liliana Demirdjian \\ lilidemir@yahoo.com.ar \\ Universidad Nacional de Buenos Aires, Argentina
}

REVCOM. Revista científica de la red de carreras de Comunicación Social

Universidad Nacional de La Plata, Argentina

ISSN: 2451-7836

Periodicidad: Bianual

núm. 11, 2020

redcom.revcom@gmail.com

Recepción: 20 Agosto 2020

Aprobación: 12 Octubre 2020

URL: http://portal.amelica.org/ameli,

jatsRepo/203/2031709001/index.html

DOI: https://doi.org/10.24215/24517836e044
Resumen: El salto tecnológico ocurrido hacia fines del pasado siglo y la aparición de nuevas modalidades de comunicación han repercutido en las dinámicas de la representación y la participación política de los regímenes democráticos. Asimismo, ha generado una configuración del espacio público multimediático que plantea la necesidad de estudiar simultáneamente medios tradicionales y digitales. Dentro de este marco general se presentan los resultados de un estudio de caso sobre el modo en que la noción de grieta circuló y se instaló en diversos escenarios mediáticos. Este trabajo asume la complejidad que subyace a la conformación del espacio público contemporáneo e intenta aportar al análisis de ciertos aspectos del entramado cultural de la política argentina en cuyo seno se desarrolló la elección presidencial de 2019.

Palabras clave: Cultura política, Comunicación, Espacio Público, Democracia.

Resumo: O salto tecnológico ocorrido no final do século passado e o surgimento de novas formas de comunicação impactaram a dinâmica de representação e participação política dos regimes democráticos. Da mesma forma, gerou uma nova configuração do espaço público multimídia que impõe uma necessidade crescente de estudar simultaneamente as mídias tradicionais e digitais. Nesse quadro geral, são apresentados os resultados de um estudo de caso sobre a forma como a noção de crack circulou e se instalou em diversos cenários midiáticos. Este trabalho assume a complexidade subjacente à conformação do espaço público contemporâneo e busca contribuir para a análise de alguns aspectos do tecido cultural da política argentina em que ocorreu a eleição presidencial de 2019.

Palavras-chave: Cultura Política, Comunicação, Espaço Público, Democracia.

Abstract: The technological leap that ocurred in the end of the past century and the emergence of new modalities of communication, have affected the dynamics of political representation and participation in the democratic regimes. In the same way, they have generated a new configuration for the multimedia public space that imposes a growing need of simultaneously studying traditional and digital media. In this general frame are introduced the results of the study case about the ways in which the rift notion has circulated and has set up in different media stages. This work assumes the complexity 


\begin{abstract}
that underlies the shaping of contemporary public space and tries to contribute to the analysis of certain aspects of the cultural framework of Argentine politics within which the 2019 presidential election took place.
\end{abstract}

Keywords: Political culture, Communication, Public Space, Democracy.

\title{
INTRODUCCIÓN
}

El pulso de la política en el seno de las democracias contemporáneas se encuentra signado, entre otros factores, por la complejidad que supone el nuevo estatuto de un espacio público en permanente proceso de transformación y en el que conviven diversos tipos de medios. La veloz evolución de las tecnologías digitales produjo una mayor densidad del tejido comunicacional en el que se gesta la actual cultura política multimediática. La nueva configuración del espacio público multimediático genera condiciones que invitan a estudiar simultáneamente medios tradicionales y digitales. A su vez, las mencionadas transformaciones han repercutido también en las dinámicas de la representación y la participación, dimensiones que resultan centrales para el estudio del funcionamiento democrático. Esta conceptualización general servirá de marco para observar el modo en que la noción de grieta circuló y se instaló en diversos escenarios mediáticos de la Argentina.

La construcción discursiva del término grieta ocupó un rol central en la cultura política massmediática (Quevedo, 1997) a partir del impulso que los medios tradicionales le dieron en los últimos años del kirchnerismo y durante toda la presidencia de Mauricio Macri (2015-2019). La palabra grieta materializó una estrategia de los medios más relevantes en cantidad de lectores (Clarín y La Nación) para conceptualizar su visión sobre el estado de cosas vigente en el país. El uso de este término suponía la existencia de una sociedad fragmentada en dos posiciones políticas aparentemente irreconciliables. De este modo, los medios replicaron sistemáticamente la versión antinómica que caracterizó la historia política argentina (Grimson, 2019). Según se observó, la noción de grieta fue propagada por los medios a instancias del interés de un sector de la clase dominante que concibió en la posibilidad de extremar la polarización del electorado una vía para acceder al poder. Este fenómeno excede el ámbito político nacional, en tanto es factible identificarlo -con diferentes matices- en diversos países de la región. Y, también, trasciende el ámbito exclusivamente político ya que es utilizado para referir a múltiples fenómenos y aspectos de la vida social.

Este trabajo intenta pues abordar, a partir del análisis de diferentes medios de prensa tradicional y de la red social Twitter, algunos rasgos del entramado cultural de la política argentina en cuyo seno se desarrolló la elección presidencial de 2019. La investigación empírica adoptó un enfoque metodológico cualitativo centrado en el análisis de marcos para el estudio de las notas recogidas en la prensa escrita y de las intervenciones de los usuarios en plataformas digitales. En este sentido, se realizó un relevamiento de prensa con el propósito de construir un corpus cuyo estudio posibilitó la posterior elaboración de datos. En el caso de la red social Twitter, el trabajo se focalizó en el seguimiento de los trending topics ${ }^{1}$ vinculados con el tema estudiado. El relevamiento de información dentro de esta red social se realizó a partir de datos recogidos de la página web Trendinalia.com. El estudio del corpus seleccionado se llevó a cabo a partir del análisis de marcos (frame analysis) y de la fijación de agenda (Paletz y Entman, 1986; Lakoff, 2006; Sadaba, 2007). En términos teóricos - metodológicos la relevancia de esta elección se fundamenta en el gran salto dentro de la teoría de la agenda que se produce con la incorporación de los conceptos de marco y enmarcado (Sadaba, 2007). En esta línea, se asume que el papel de los medios masivos de comunicación "es socializar a la gente para que acepte la legitimidad del sistema político de su país (...) dirigir sus opiniones de modo que no socaven y a menudo apoyen los objetivos internos y externos de las élites" (Paletz y Entman, 1986, p.103). Este tipo de análisis 
empírico permite indagar la forma en que los medios producen determinadas construcciones simbólicas de la realidad, cuya influencia en asuntos y actores concretos, modelan la cultura política a lo largo del tiempo.

\section{DEMOCRACIA, PARTICIPACIÓN Y REPRESENTACIÓN: ENTRE MEDIOS TRADICIONALES Y REDES SOCIALES}

Las transformaciones que se producen a partir del uso de las redes sociales permiten identificar continuidades y rupturas en el orden de la representación política. En esta línea se evidencian, básicamente, dos visiones contrapuestas sobre las nuevas condiciones en las que tiene lugar el vínculo representativo. Por una parte, y como un dato singular dentro del nuevo escenario que se abre con el uso de las redes sociales, resulta plausible sugerir que éstas contribuyen a disminuir la distancia entre representantes y representados. Se considera que existen mayores posibilidades para interactuar y seguir en directo el minuto a minuto de la vida de los políticos y de estos para relacionarse con la ciudadanía. Por otra parte, también es posible interpretar que la distancia entre representantes y representados no necesariamente se acorta como resultado del uso de las nuevas tecnologías. La realidad es que los políticos habitualmente no manejan sus redes, no son ellos quienes se encuentran del otro lado de sus teléfonos celulares interactuando con el ciudadano común. Pocos desconocen hoy que son sus responsables de prensa quienes les manejan las redes sociales. Se trata de consultoras y de empleados a contrato dedicados a diseñar la forma y el contenido de la información que circula sobre cada candidato en épocas de campaña y, luego, sobre la gestión de aquellos que logran convertirse en representantes electos. Hasta aquí, se observa que existe cierta dosis de controversia en relación con las nuevas condiciones y posibilidades en virtud de las que se desarrolla el vínculo representativo como resultado del uso de las redes sociales. Con independencia de la valoración que se formule sobre el uso de estas últimas, un aspecto resulta ineludiblemente novedoso: gracias a la comunicación vía redes sociales los políticos pueden, cada vez más, llegar a la ciudadanía sin la mediación de los medios tradicionales de comunicación. Así, el papel de estos últimos y en su seno de la labor periodística, aparecen, en mayor o menor medida, resignificados. En efecto, en la actualidad, quienes se desempeñan en los medios masivos se ven en la necesidad de referir permanentemente a la actividad de los políticos en las redes sociales ${ }^{2}$. De hecho, algo queda claro en esta nueva etapa: se ha roto el monopolio informativo que históricamente se encontraba en manos de los medios tradicionales (prensa, radio, televisión). De ahora en más la discusión, en todo caso, girará en torno a cuál es el grado de ruptura que se produjo y cuántas posibilidades existen de que dicha ruptura se profundice. Varios son, en rigor, los planos en los que se observan transformaciones: la actividad periodística cuenta con nuevos recursos para producir información y, al mismo tiempo, se modifican las formas de consumirla. Para describir el carácter de la actividad que se desarrolla en esta avenida de doble mano en la que se produce el ida y vuelta entre la producción y el consumo de información se ha llegado a sugerir la noción de prosumidor ${ }^{3}$ (Toffler, 1981). El mismo apunta a describir una nueva realidad en la que los ciudadanos, gracias a la tecnología disponible, no solo tienen la capacidad de diversificar sus posibilidades de consumo, sino también de ampliar su capacidad de producir y distribuir información.

Por su parte, la dimensión de la participación política también se ve interpelada por el advenimiento de las tecnologías digitales en el campo de la comunicación. Nuevos modos de activismo se desarrollaron como resultado de las posibilidades que brindan los instrumentos de la Web 2.0. En diferentes puntos del planeta han tenido lugar movimientos sociales surgidos de las redes. El impacto político de sus demandas les otorgó visibilidad y posibilitó el conocimiento público de sus reclamos a nivel global. Tal es el caso de la denominada Primavera Árabe, iniciada con las revueltas que tuvieron lugar a partir de 2010 en Egipto y que se trasladaron luego a otros países de la región (como Túnez, Turquía, Siria y Marruecos). También en el mundo occidental acontecimientos como el 15M en España, el Occupy Wall Street en Estados Unidos, los Chalecos Amarillos en Francia, o más recientemente en Sudamérica Chile despertó y el movimiento Ele não en Brasil resultan buenos ejemplos de las potencialidades que ofrecen redes sociales como Facebook $o$ 
Twitter. En efecto, el activismo en las redes ha sido útil para expresar tanto el descontento ciudadano frente a regímenes autoritarios como también frente a casos de corrupción por parte de la clase política. En ambos casos, los medios masivos recogieron, visibilizaron y amplificaron a nivel global los mencionados movimientos de protesta. Según Joan Subirats (2015) los nuevos movimientos sociales, usuarios de nuevas tecnologías digitales, debieron comprender que atender a la relación con los medios de comunicación tradicionales resultaba un aspecto central para sus intereses ya que «el éxito o el fracaso de las protestas o acciones está condicionada por el interés que muestren los medios sobre ella» (p. 125). Aquí radica, entre otras, una de las razones por las cuales es necesario atender al estudio de ambos sistemas de medios. A su vez, junto con la circulación de mensajes que se producen en estos dos sistemas de comunicación no se debe perder de vista que pervive, con mayor o menor fuerza según el caso, la acción de la movilización popular en el espacio público. Esto supone un complejo escenario para el abordaje de la dimensión política que, en parte, ya observara en nuestro país María Cristina Mata (1992). Hace más de un cuarto de siglo, cuando aún no existían las redes sociales, Mata se refería a la relación entre política y televisión y sugería: «Lo que desafía nuestra comprensión y nuestro juicio al asumir que la política se construye hoy en esa tensión entre la plaza y la platea y no en virtud de una lisa y total destrucción de la primera bajo el imperio de la segunda, es la cesión que ello implica, el sometimiento de todo aquello por lo que la plaza convoca a la necesidad y al placer de verse» (1992, p. 74). Ese placer de verse, de encontrarse pareciera reeditarse hoy a partir de la presencia en las redes. Es por ello que Facebook, tal como lo describió su fundador Mark Zuckerberg, pretende constituirse como un generador de infraestructuras sociales que les permita a sus usuarios la construcción de una comunidad mundial «adecuada para todos» (citado en Klinenberg, 2019). Este rol que Facebook intenta representar se asemeja notablemente a los que en otros tiempos cumplieron grupos sociales como las iglesias o los sindicatos en tanto «brindan razones de ser y esperanza, la validación moral de nuestra pertenencia a algo más grande; el consuelo de saber que no estamos solos y que una comunidad nos cuida» (Klinenberg, 2019). A pesar de ello, es importante no perder de vista que, en redes de este tipo (Facebook, Twitter, Instagram), la instancia del encuentro o comunidad resulta de mero carácter virtual. Luego, solo en ocasiones como las anteriormente mencionadas, los diversos tipos de colectivos y de movimientos que surgen en esa nueva ágora virtual se trasladan a las calles. Suele ser, entonces, la ocupación del espacio público urbano la que les otorga la capacidad de modificar en alguna medida el escenario que los condujo a la protesta. Así, el pulso de la política en el seno de las democracias contemporáneas se encuentra signado -entre otros factores- por la complejidad que supone el nuevo estatuto de un espacio público en permanente proceso de transformación.

Finalmente, en términos amplios resulta interesante destacar que, tal como habitualmente sucede con la llegada de cada nueva tecnología, se pueden identificar dos visiones enfrentadas en torno a las consecuencias derivadas del uso de las redes sociales. Dichas visiones las encarnan autores (Porras y Araya, 2003; Rodríguez, 2019; Alonso Muñoz, 2015) que han sido caracterizados como ciberoptimistas o ciberescépticos ${ }^{4}$. Los primeros apuestan a que el uso de estas tecnologías brindará mayores chances de generar un escenario de democratización, es decir, que contribuirá a fortalecer las posibilidades de participación y a facilitar la deliberación. Los segundos, en cambio, no manifiestan ninguna esperanza respecto a que las nuevas tecnologías ahora disponibles sean portadoras, en sí mismas, de un espíritu democratizador. Esta distinción reedita la que planteara Umberto Eco (1985) en su clásica obra Apocalípticos e integrados. En ella, el semiólogo italiano presentaba dos visiones contrapuestas en torno a las consecuencias sociales que se podían esperar como resultado de la generalización de la televisión.

\section{NOTAS SOBRE UN ESTUdIO DE CASO: EL USO DEL TÉRMINO GRIETA EN LOS MEDIOS TRADICIONALES Y EN LAS REDES SOCIALES}

Este último apartado se propone una aproximación al estudio de la aparición de un término que forma parte central de nuestra «cultura política massmediática reciente» (Quevedo, 1997). Según se observó, 
el término grieta se convirtió en la principal noción a partir de la que los medios masivos presentaron el devenir político nacional en los últimos años del kirchnerismo y durante toda la presidencia de Mauricio Macri. Como se anticipó, en el seno de una sociedad hipermediatizada (Carlón, 2016, p. 140) un análisis consistente requiere considerar la aparición del mencionado término tanto en los medios tradicionales como en los nuevos medios. Se establecerá, entonces, una comparación sobre la utilización del término durante el primer semestre del 2019 en los medios tradicionales de prensa gráfica y en la red social Twitter, por ser ellos importantes formadores de opinión política. En 2019 tuvo lugar la última elección presidencial en Argentina y la instancia que permitió sustanciar las fórmulas de los partidos que compitieron a nivel nacional en el mes de octubre se dio en las elecciones Primarias Abiertas Simultáneas y Obligatorias (PASO) del día 11 de agosto de ese año.

El análisis empírico de un corpus de prensa escrita formado por las noticias que incluyeran el término grieta durante el primer semestre de 2019 de los periódicos Clarín y La Nación y la red social Twitter permitió observar que esta fue la palabra privilegiada con la que se intentó definir desde los medios estudiados el clima político que, supuestamente, imperaba en la sociedad y, especialmente, en el seno de la clase política local. Es decir, se trató de una estrategia de los medios para conceptualizar el estado de cosas vigente en el país. Esta lectura de la realidad propuesta por los medios fue central desde el inicio del gobierno de Cambiemos en 2015. Tal lectura resulta coincidente con el discurso del presidente Mauricio Macri, quien apuntó durante todo su mandato a establecer una línea tajante entre la gestión kirchnerista y la propia, aludiendo a un pasado nefasto al cual caracterizó como una "pesada herencia”. Según afirma Alejandro Grimson (2019), esta «caracterización que resultó necesaria y útil a los efectos de justificar la implementación de una política económica de profundos ajustes» (p. 314). En palabras de Horacio González, la grieta «es un concepto televisivo para analizar la política» (La Nación, 15-05-2019). Sobre este punto interesa destacar el carácter performativo que posee el lenguaje (Austin, 2003). Especialmente porque el uso que se realizó del término grieta tuvo un carácter de esta índole. La generalización de su uso en los medios tradicionales produjo, por sí misma, un efecto legitimador de una determinada lectura de la realidad. Toda lectura sobre la realidad es, en tanto tal, siempre subjetiva y expresa el punto de vista de quienes la narran. Para instalar la noción de grieta, como resulta habitual, los medios hicieron uso del blindaje que le proporciona el espacio de la enunciación periodística. Espacio que se autoproclama objetivo y neutral pero tras el cual se desarrolló, por cierto, una trama de responsabilidades que le permitió establecer víctimas y victimarios. Un ejemplo muy explícito al respecto lo constituye la nota de opinión publicada por el periodista Luis Majul en el diario La Nación el 5 de diciembre de 2013, cuyo título anuncia: «A la grieta la instalaron Néstor y Cristina».

En este sentido, es posible afirmar que «la grieta, o fractura entre dos sectores radicalmente opuestos, ha sido un factor recurrente en el desarrollo histórico nacional, que se ha cristalizado en múltiples relaciones políticas dicotómicas. Desde la puja entre la tradición liberal y la nacional-popular, fue adquiriendo diversos nombres: unitarios y federales, peronistas y gorilas, yrigoyenistas y antipersonalistas, entre otros» (Demirdjian, 2020). Por tanto, es factible pensar que la cuestión de la grieta no remite a un modelo de democracia en particular, sino que se trata de una noción cuya potencia radica en su capacidad de calar profundo en el entramado sociopolítico, independientemente del período histórico y del régimen de gobierno vigente de cada una de las etapas por las que atravesó la conformación del estado nacional.

La primera mención del término en un lugar de relevancia dentro de la prensa local la realizó el periodista Jorge Lanata cuando era director de Página/12 en el año 1989 (Demirdjian, 2019). Si bien Lanata se deslinda de la responsabilidad de haber instalado el uso del término dentro del periodismo ${ }^{5}$, ha sido no solo su mentor sino también uno de sus principales divulgadores. Más recientemente se puede rastrear la reaparición del término hacia 2008 cuando se produce el conflicto entre el gobierno kirchnerista y el campo por la aplicación de retenciones al sector agrario $^{6}$ (Grimson, 2019, pp. 284-290). En la prensa gráfica el término comienza a ser recurrente hacia el año 2010. A partir de allí, su uso tuvo un incremento sostenido ${ }^{7}$. Una primera observación sobre el uso de la palabra grieta permite afirmar que, en el discurso de los medios, apareció 
asociada al kirchnerismo ${ }^{8}$. Según la historia que construyen los medios de mayor audiencia, la grieta tuvo su origen en el gobierno de Cristina Fernández de Kirchner. De acuerdo con esta versión, se le atribuyó a la figura de la expresidenta la responsabilidad de haber sido su gran arquitecta, construyendo una línea divisoria que partió en dos a la sociedad argentina. A partir del año 2015 los medios replicaron sistemáticamente la versión antinómica que comenzaba a plantear el nuevo gobierno. La fórmula fue exitosa y, de allí en más, la idea de que existía una grieta fue preponderante para hacer inteligible la vida política nacional. El término se instaló dentro del lenguaje corrientemente utilizado en los medios y evidenció sus puntos más altos de circulación en los años electorales en los que, como se anticipó, se la utilizó con la intención de polarizar a la ciudadanía llamada a votar' ${ }^{9}$ La grieta supone una divisoria de aguas, alude a una situación de confrontación radical, parte del supuesto de que existen en la sociedad dos partes enfrentadas de manera aparentemente irreconciliable. La grieta fue presentada por los medios como si fuera un diagnóstico sobre la salud de la Nación, un síntoma que si no se resuelve acarrea pésimos pronósticos para el conjunto del país. Sin embargo, en tanto que actores conscientes de las graves consecuencias que su uso implicaba en ningún momento se abstuvieron de reproducirla. Antes bien, la incentivaron. Así, cuando se presenta la fórmula presidencial Fernández-Fernández en mayo de 2019, la prensa se vio descolocada y una parte de ella interpretó la decisión de Cristina Fernández de Kirchner de convertirse en candidata a vice presidenta como una estrategia segura para llegar al poder ${ }^{10}$. Según se comentó en diversos medios, se trataba de una decisión que apuntaba a romper la polarización del electorado. Como se puede observar a continuación, un mes antes de las elecciones generales, y en conocimiento de los resultados de las elecciones primarias (PASO), la Revista Barcelona publica la siguiente tapa:

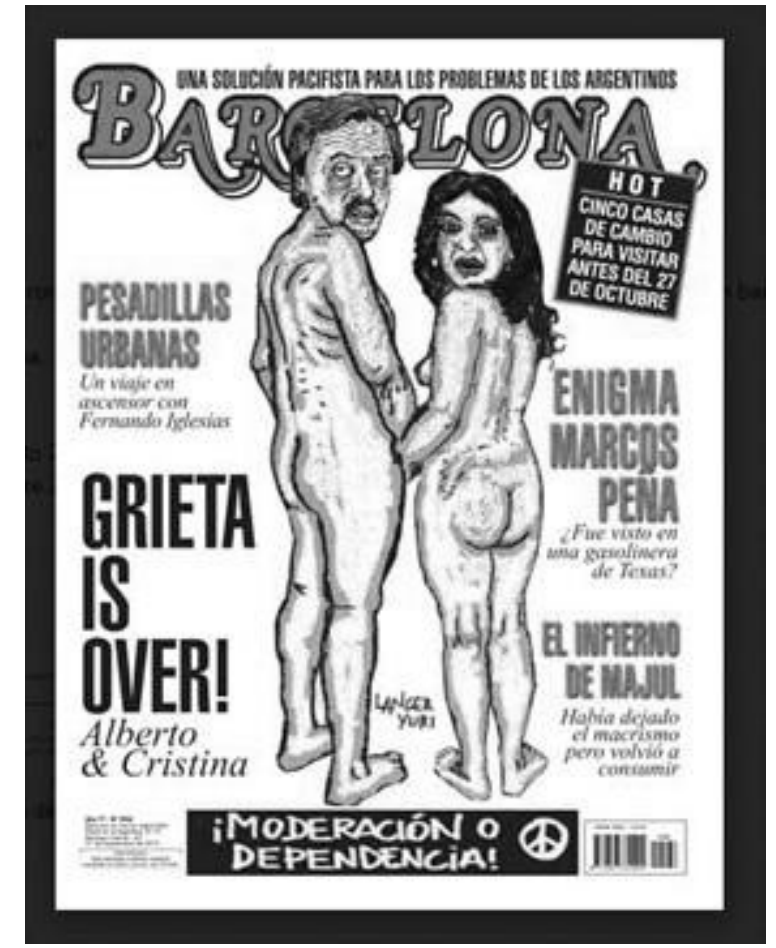

FIGURA 1

Tapa de Revista Barcelona, 27 de septiembre de 2019.

Por su parte, el principal programa político de Canal 13 (Grupo Clarín) presentó al candidato Alberto Fernández como un títere de Cristina Fernández de Kirchner. La sitcom Argentina: tierra de rencor y venganza, transmitida en el programa Periodismo Para Todos conducido por Jorge Lanata, lo presentaba como una marioneta manejada por la ex presidenta (Demirdjian, 2019) 
Los medios utilizaron el término grieta inicialmente en los programas de periodismo político. No obstante, a partir del 2015 su uso se convierte en una clave para referir hechos que tienen lugar en muy diversos campos de la vida social. El amplio espectro dentro del cual el periodismo utilizó el término incluyó, entre otros, los ámbitos de la economía, el espectáculo y el deporte.

\section{Diario Clarín}

$$
20
$$

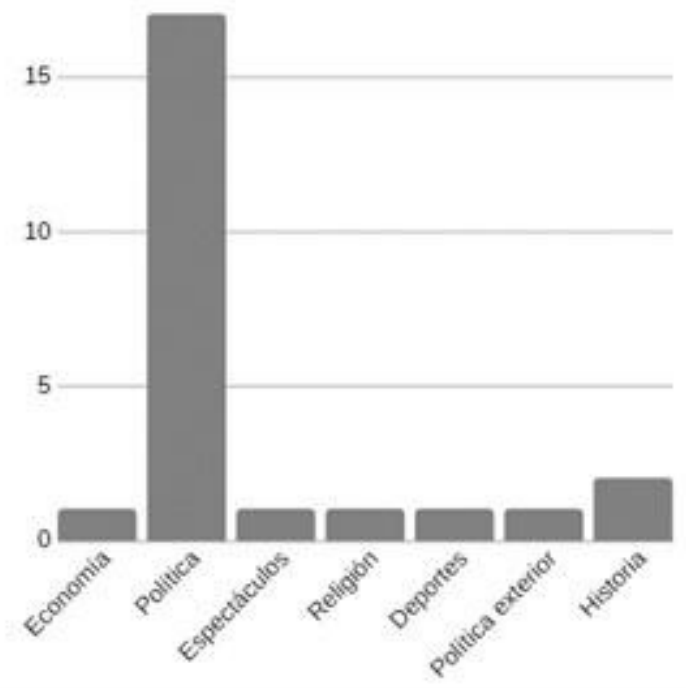

GRÁFICO 1

Distribución del uso del término grieta según temáticas en titulares del diario Clarín en el segundo bimestre 2019 (abril y mayo)

Fuente: elaboración propia 


\section{Diario La Nación}

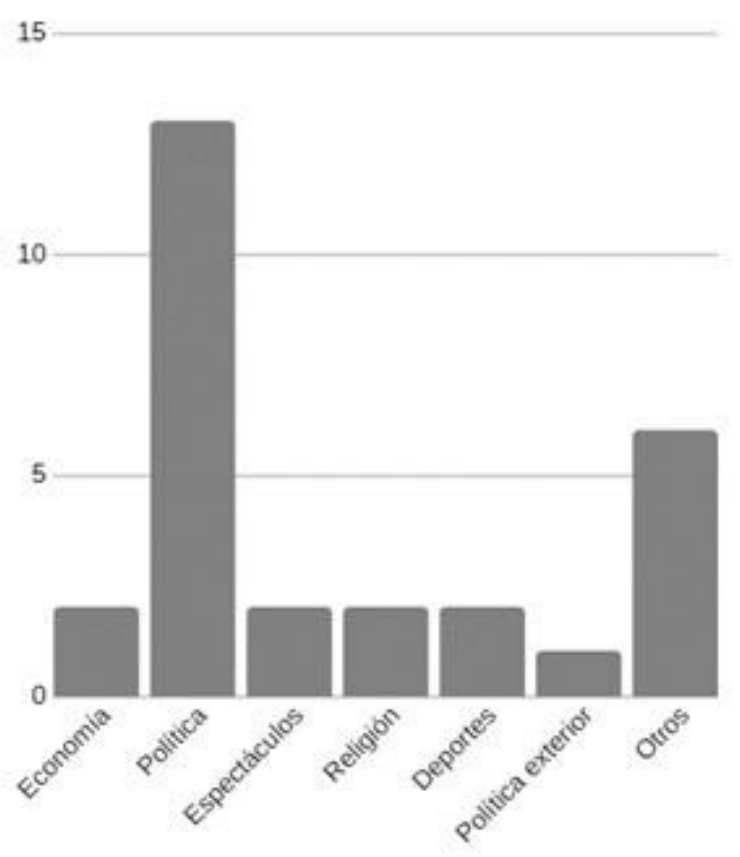

GRÁFICO 2

Distribución del uso del término grieta según temáticas en titulares del diario La Nación en el segundo bimestre 2019 (abril y mayo).

Fuente: elaboración propia

El periodismo hizo extensivo el uso del término a las redes sociales, tal como lo expresan el texto y la imagen publicada en la cuenta de Twitter del diario Olé que se presentan a continuación. En este tweet se retrata el conflicto entre el futbolista Mauro Zárate, actual jugador del equipo Boca Juniors, y su equipo anterior (Club Atlético Vélez Sarsfield). 


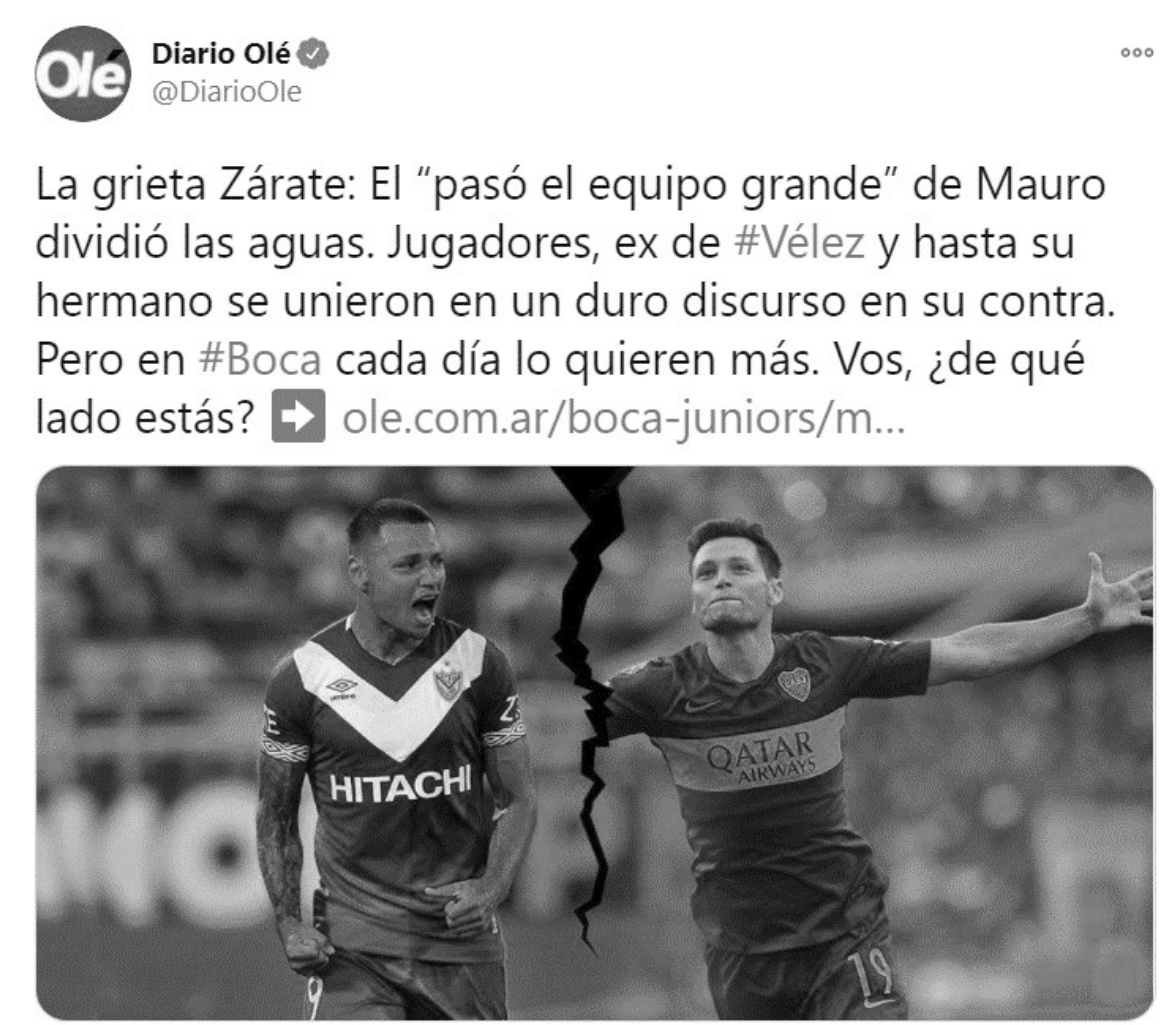

9:21 p. m. 17 may. $2019 \cdot$ TweetDeck

\section{FIGURA 2}

Publicación de Twitter del diario Olé

Fuente: elaboración propia

El éxito de la rigurosa labor desarrollada por los medios para imponer la idea de que existía una grieta se puede observar en el modo en que se propagó el término más allá del espacio estrictamente mediático. La palabra grieta ingresó en el discurso de varios miembros de la clase política, que lo incluyeron en sus análisis sobre la coyuntura nacional. Simultáneamente se puede observar su uso en la actividad de varios dirigentes políticos en las redes sociales. Un ejemplo muy ilustrativo sobre este punto es el de Roberto Lavagna -ex ministro de Economía durante el gobierno de Néstor Kirchner y candidato a presidente en las elecciones de 2019 por la alianza Consenso Federal- en Twitter, quien escribió desde su cuenta @RLavagna: «Por el camino de la COHERENCIA y el CONSENSO seguimos trabajando junto a @MiguelLifschitz y @Stolbizer por un futuro mejor para todos los argentinos. La única vía para ganarle a la grieta». Este mensaje, expuesto en la siguiente imagen fue, a su vez, retwitteado por Margarita Stolbizer. 


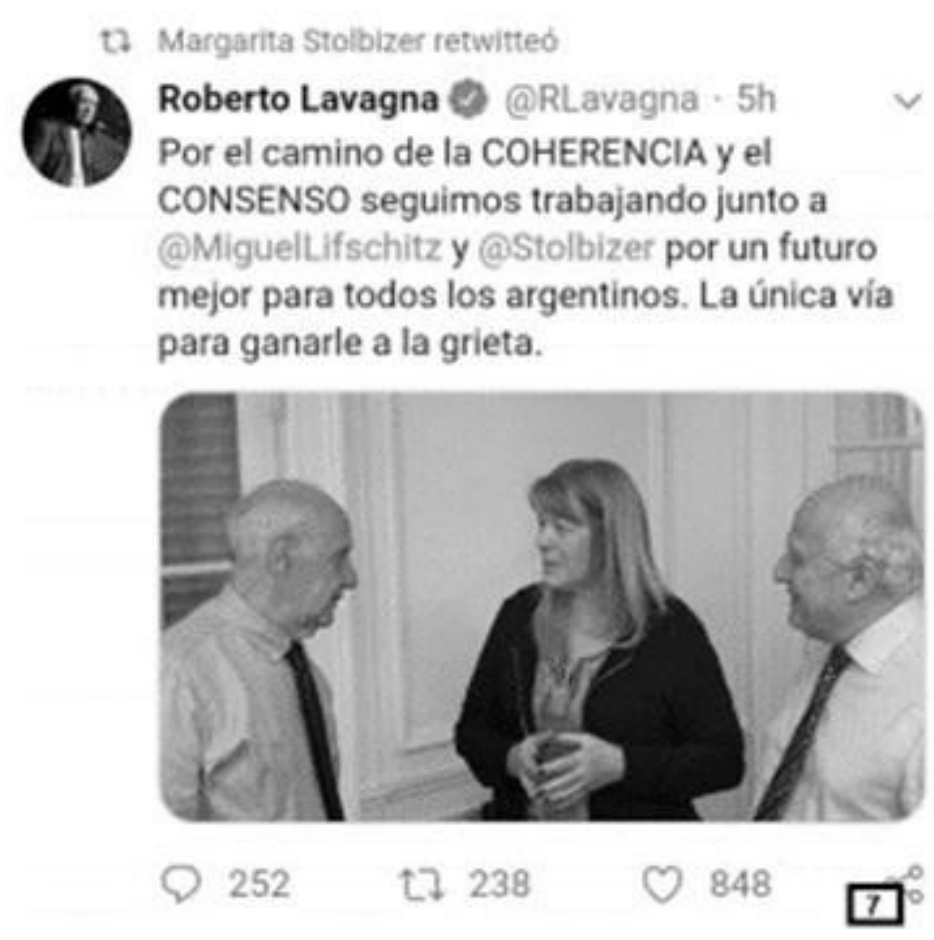

FIGURA 3

Tweet de Roberto Lavagna

Fuente: elaboración propia

En virtud de la instalación en la agenda política de la idea de que existía una grieta, su alusión caló tan profundo que, en el intento por revertir sus efectos, fue recogida incluso por referentes del espacio kirchnerista. Al respecto, se menciona el título del libro publicado en 2019 por el candidato a gobernador de Buenos Aires por el Frente de Todos y ex ministro de economía de Cristina Fernández de Kirchner, Axel Kicilloff: Y ahora, ¿qué? Desengrietar las ideas para construir un pais normal ${ }^{11}$

Un dato adicional que surge del análisis de prensa realizado sobre los diarios Clarín y La Nación señala la presencia de una dicotomía en el discurso de los medios entre la idea de grieta y de consenso. Como se sugirió ya, la grieta se asocia con la figura de Cristina Fernández de Kirchner, mientras que la figura de Mauricio Macri durante los primeros meses del 2019 es regularmente asociada a situaciones de consenso. 


\section{Diario Clarín}

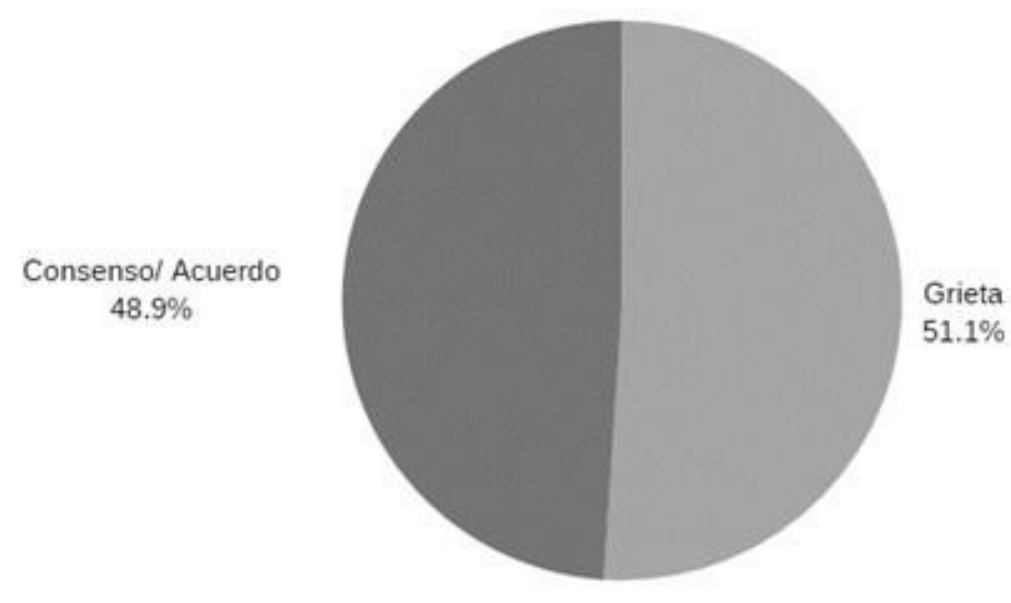

GRÁFICO 3.

Mención de los términos grieta y acuerdo/consenso en los titulares del diario Clarín en el segundo bimestre 2019 (abril y mayo).

Fuente: elaboración propia

\section{Diario La Nación}

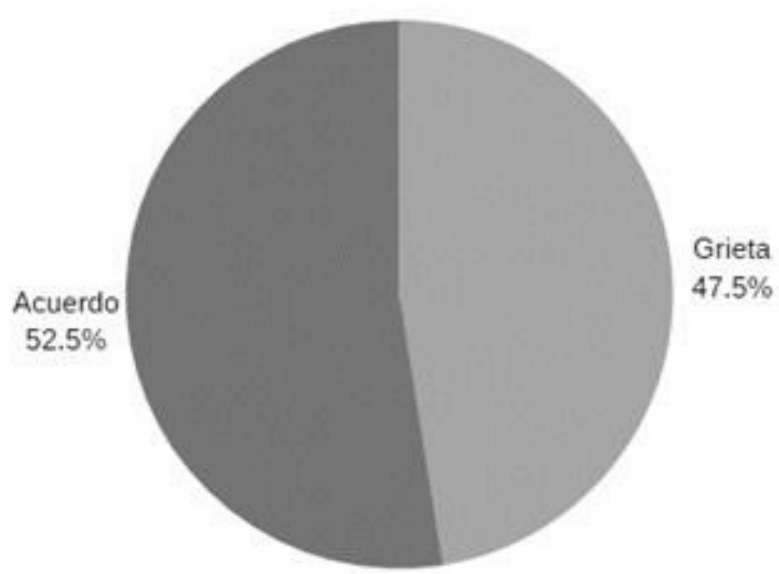

GRÁFICO 4

Mención de los términos grieta y acuerdo en los titulares del diario La Nación en el segundo bimestre 2019 (abril y mayo).

Fuente: elaboración propia

Un rasgo de la cultura política massmediática reciente a nivel nacional, pero también en el orden más amplio del contexto regional, consiste en trabajar sobre la noción de grieta. Un ejemplo de ello es la declaración que realiza en Venezuela el dirigente opositor Leopoldo López cuando el 2 de mayo de 2019 dejó su arresto domiciliario y se mostró junto a Juan Guaidó. En esa ocasión hizo una declaración que, en Argentina, reprodujo el noticiero de Canal 13 de Buenos Aires en su edición de medianoche y que se cita 
aquí según publicara textualmente el diario La Nación: «La “operación libertad” involucra a todos. Recibí un indulto del presidente Guaidó. El quiebre ha comenzado. La fisura que comenzó el 30 de abril se convertirá en una grieta que romperá la represa» (La Nación, 2-5-2019).

En resumen, se ha intentado evidenciar el modo en que el término grieta remite a una idea de división radical de la sociedad propagada por los medios a instancias del interés de un sector de la clase dirigente que observó en la posibilidad de polarizar al extremo al electorado una vía para acceder al poder. Este fenómeno, que excede el ámbito político nacional, se manifestó con diferentes matices en diversos países de la región ${ }^{12}$. Así también es posible identificarlo en Estados Unidos. Desde el inicio de la era Trump una parte importante de la prensa estadounidense comenzó a trabajar sobre la idea de la polarización y resulta habitual en sus páginas la inclusión del término rift. ${ }^{13}$

En paralelo, interesa señalar un primer resultado del seguimiento realizado durante los seis meses iniciales de 2019 sobre los diez primeros temas que fueron tendencia en la red social Twitter. El seguimiento se realizó a partir de los datos aportados por el sitio Trendinalia.com y evidenció que la utilización del término grieta en esta red social no fue en absoluto relevante entre esos diez primeros trending topics (TT), resultado que dista en mucho de lo observado anteriormente en los medios tradicionales. En primer término, cabe señalar que la noción de grieta propagada por dichos medios no tuvo un efecto inmediato, cuando menos, como tema explícito dentro de los hashtags que encabezaron los TT durante el primer semestre del año. En rigor, el relevamiento realizado sobre la red social Twitter ha permitido identificar que los principales temas que tuvieron lugar en la franja de los diez primeros TT se concentraron en cuestiones de muy diverso orden pero que, mayormente, no se vinculaban con cuestiones de carácter político. En lo fundamental, los primeros diez TT se relacionan con programas televisivos, eventos deportivos, figuras mediáticas, etc. En relación con este punto, entonces, se observa que la comunicación en las redes sociales replica las prácticas de banalización y espectacularización de la política que se han generalizado en el sistema de medios tradicionales. Asimismo, sería relevante establecer qué influencia tienen esa clase de prácticas en relación con las condiciones de posibilidad y con los modos en que, finalmente, se desarrolla el debate político en este nuevo ágora virtual.

\section{Conclusiones}

En principio, a partir de los resultados obtenidos, cabe señalar que resulta de interés continuar explorando si se sostiene la tendencia a la banalización y espectacularización de la política en el espacio público virtual. En todo caso, la corroboración de esta tendencia en el tiempo abonaría la posición de aquellos autores que se inscriben en la perspectiva ciberescéptica. Del seguimiento realizado se puede aproximar un primer señalamiento: el uso de las redes sociales no estaría, en principio, aportando al intercambio de información sobre asuntos relacionados con la gestión pública, ni ampliando las efectivas posibilidades de discusión sobre problemas comunes, ni alentando espacios de deliberación que puedan promover una mejora del debate público.

Finalmente, es posible afirmar que el uso de medios digitales en contextos de campañas políticas constituye un factor destacado en los procesos electorales de la región. En el caso argentino, la campaña presidencial que llevó al gobierno a Mauricio Macri en 2015 estuvo fuertemente apoyada en las redes sociales. El mismo mecanismo de campaña política se aplicó durante las elecciones de 2017 en Ecuador. Más recientemente, en 2018 en Brasil, la llegada de Jair Bolsonaro al Planalto también ha sido atribuida, entre otros factores, a la campaña que se desarrolló en las redes sociales y a la novedad de la inclusión del WhatsApp como medio privilegiado de circulación de mensajes de propaganda (Chomsky, 2002; Wolf, 1996; Rodríguez Fernández, 2019). En resumen, las redes sociales fueron protagonistas de gran parte de los slogans y spots de campaña, noticias falsas y contenidos en torno al posicionamiento de los principales candidatos políticos.

Las democracias contemporáneas, como se sugirió, se encuentran extremadamente mediatizadas e insertas en un permanente proceso de transformación que profundiza las dinámicas de modificación del espacio 
público y de la vida política. Este nuevo escenario se configura sobre la base de un espacio público que, al tiempo que es colonizado por grandes corporaciones, abre posibilidades sobre la base de la proliferación de redes sociales para la difusión y presentación de ideas y posicionamientos políticos-ideológicos de diversos sectores de la sociedad civil. Es un hecho de público conocimiento que existe una creciente administración y organización de estas posiciones ideológicas, y que los procedimientos de disposición y control dentro de las redes resultan cada vez más habituales. En términos tradicionales, se sugirió que los medios masivos tenían una determinada incidencia en la formación y dominio de la opinión. Frente a ello se pensó que las redes sociales podían constituir ámbitos propicios para la libre expresión y circulación de ideas y propuestas, ajenos a cualquier tipo de injerencia y control. En este sentido, fueron propuestas como una instancia que podía garantizar el ideal de transparencia y de calidad informativa. En la actualidad, es evidente el éxito de la proliferación de dispositivos y programas desarrollados para intervenir y orientar la formación de opinión en las redes.

Provisoriamente y en función de lo hasta aquí señalado, se puede corroborar que la comunicación política funciona hoy en un territorio definitivamente más complejo que aquel que se definía en los medios tradicionales y que ello obliga a un abordaje que incluya el estudio de diversos escenarios mediáticos. Sumado a todo esto es importante no perder de vista que el orden comunicativo -que tiene lugar a partir de la circulación de mensajes en las redes y que funciona sobre la base de múltiples plataformas y recursos tecnológicos- originan nuevos problemas de estudio. Sin duda, los esfuerzos deberán ser mayores para tornar inteligible el nuevo escenario comunicacional signado por diversos regímenes de visibilidad, además del televisivo.

Los modos de control y manipulación de la comunicación resultan infinitamente más sutiles y efectivos en esta nueva era gobernada por el big data y por el uso cada vez más corriente de las noticias falsas. Para finalizar, si bien se observa la emergencia de nuevas formas de resistencia y organización social, un primer balance parece darle crecientes razones a los diagnósticos y argumentos esgrimidos por quienes adscriben a la perspectiva ciberescéptica.

\section{Agradecimientos}

Este trabajo es resultado de la labor desarrollada en el marco de los proyectos de investigación que la autora codirige y dirige en el seno de la UBA y de la UNTREF, respectivamente. El mismo surge de una reelaboración de la ponencia presentada a la mesa de "Los desafíos actuales de las Ciencias de la Comunicación como espacios de construcción de conocimientos y prácticas sociales" del $3^{\circ}$ Congreso Nacional de Sociología de la UNSJ. Este artículo incluye pues una serie de discusiones mantenidas en el desarrollo de la misma y al interior de los mencionados proyectos de investigación, en cuyo marco la autora agradece especialmente la contribución de Leandro Soto y de Florencia Iglesias.

\section{ReFERENCiAS}

Alonso Muñoz, L. (2015). Redes sociales y democracia. Una aproximación al debate sobre una relación compleja. Fòrum de recerca, (20), 557-569.

Antonio, J. (1996) ¿Y ahora qué? Buenos Aires, Argentina: Ediciones Verum Et Militia.

Aronson, P. (2007). Notas para el estudio de la globalización. Un abordaje multidimensional de las transformaciones sociales. Buenos Aires, Argentina: Biblios.

Austin, J. (2003). Cómo hacer cosas con palabras: palabras y acciones. Buenos Aires, Argentina: Paidós.

Carlón, M.(2016). Una apropiación contemporánea de la teoría de la comunicación de Eliseo Verón. En: E. Vizer y C. Vidales (coords.): Comunicación, campo(s) teorias y problemas. Una perspectiva Internacional. Salamanca, España: Comunicación social. 
Chomsky, N. (2002). La propaganda y la opinión pública. Conversaciones con David Barsamian. Barcelona, España: Crítica.

Demirdjian, L. (2019). De la democracia a la mediocracia. Nociones para abordar la política contemporánea. XIII Jornadas de Sociología. Facultad de Ciencias Sociales, Universidad de Buenos Aires, Buenos Aires.

Demirdjian, L. (2020). Cultura política massmediática. El origen de la grieta. Question/Cuestión, 1(65), e243.

Eco, U. (1985). Apocalipticos e integrados. Barcelona, España: Lumen.

Entman, R. M. y Paletz, D. L. (1986). Aceptar la legitimidad del sistema. En D.A. Graber (Comp.), El poder de los medios en la política (pp. 103-111). Buenos Aires, Argentina: Grupo Editor Latinoamericano.

Fernández, J. (Coord.). (2014). Postbroadcasting. Innovación en la industria musical. Buenos Aires, Argentina: La Crujía.

Fernández de Kirchner, C. (2019). Sinceramente. Buenos Aires, Argentina: Sudamericana.

Grimson, A. (2019). ¿Qué es el peronismo? De Perón a los Kirchner, el movimiento que no deja de conmover a la política argentina. Buenos Aires, Argentina: Siglo XXI.

Horacio González: "Venezuela no es una dictadura y Maduro es víctima de un golpe de Estado". (15 de mayo de 2019). La Nación. Recuperado de: https://www.lanacion.com.ar/politica/horacio-gonzalez-venezuela-no-es-dictadura -maduro-nid2248145

Klinenberg, E. (abril 2019). Facebook contra los espacios públicos. Le Monde Diplomatique. Edición (238)

Leopoldo López: "No quiero volver a la cárcel, pero no le temo". (2 de mayo de 2019). La Nación. Recuperado de: ht tps://www.lanacion.com.ar/el-mundo/leopoldo-lopez-no-quiero-volver-carcel-pero-nid2243766

Lakoff, G. (2006). No pienses en un elefante. Lenguaje y debate politico. Madrid: Ediciones Península.

Lois, I. y Wainer, L. (Comp.). (2019). Por otros medios. Comunicación y golpes en América Latina. Buenos Aires, Argentina: Ediciones del CCC.

Majul, L. (5 de diciembre de 2013). A la grieta la instalaron Néstor y Cristina. La Nación. Recuperado de: https://w ww.lanacion.com.ar/opinion/a-la-grieta-la-instalaron-nestor-y-cristina-nid1644728/

Mata, M. (1992). Entre la plaza y la platea. En: H. Schmucler y M.C. Mata (coords.), Politica y comunicación ¿Hay un lugar para la política en la cultura mediática? (pp. 61-76). Buenos Aires, Argentina: Catálogo.

Natanson, J. (abril 2019). Devorados por la grieta. Le Monde Diplomatique. Edición (238).

Paletz, D. L. y Entman, R. M. (1986). Aceptar la legitimidad del sistema. En: D. Graber (comp.) El poder de los medios en la política (pp. 103-111.). Buenos Aires, Argentina: Grupo Editor Latinoamericano,

Porras, J. y Araya, R. (Eds.). (2003). E-democracia. Retos y oportunidades para el fortalecimiento de la participación ciudadana y la democracia en la sociedad de la información. Santiago, Chile: Universidad Bolivariana.

Quevedo, L. (1997). Videopolítica y cultura en la Argentina de los noventa. En Winocur, R. (Coord.), Culturas politicas a fin de siglo. México: Juan Pablos Editor-FLACSO.

Rodríguez Fernández, L. (2019) ¿Cuánto ha influido la propaganda digital en las elecciones? The Conversation. Recuperado de: https://theconversation.com/cuanto-ha-influido-la-propaganda-digital-en-las-elecciones-1269 79

Rodríguez, P. (2019). Las palabras en las cosas. Saber, poder y subjetivación entre algoritmos y biomoléculas. Buenos Aires, Argentina: Editorial Cactus.

Ruiz del Ferrier, M. y Tirenni, J. (2006). El desarrollo en la democracia de opinión. Del liderazgo político a la concentración de la propiedad mediática. En: D. García Delgado, y L. Nosetto, L. (Comps.). El desarrollo en un contexto posneoliberal. Buenos Aires, Argentina: Ciccus/Flacso.

Sadaba, T. (2007). Framing: el encuadre de las noticias. El binomio terrorismo-medios. Buenos Aires: La Crujía.

Subirats, J. (2015). Todo se mueve. Acción colectiva, acción conectiva. Movimientos, partidos e instituciones. Revista Española de Sociología (24), 123-131.

Stiletano, M. (24 de mayo de 2019). Jorge Lanata empieza la temporada final de PPT: "En la Argentina no hay una grieta penal". La Nación. Recuperado de: https://www.lanacion.com.ar/espectaculos/jorge-lanata-nid2251266 
Toffler, A. (1981). La tercera ola. México: Edivisión

Wolf, M. (1996). La investigación de la comunicación de masas. Critica y perspectivas. Barcelona: Paidós.

\section{Notas}

1 Trending topics refiere a aquellas palabras y/o frases más repetidas por los usuarios de Twitter en un período de tiempo determinado

2 Al respecto, un dato no menor es que, de cara a las elecciones presidenciales de 2019 en Argentina, tanto Cristina Fernández de Kirchner como Mauricio Macri anunciaron sus fórmulas presidenciales por Twitter (la fórmula Fernández-Fernández se anunció el 18 de mayo y la fórmula Macri-Pichetto el 11 de junio de 2019). Ambos anuncios generaron un efecto sorpresa y les restaron a los medios masivos la posibilidad de construir con cierta antelación cualquier tipo de acontecimiento respecto al lanzamiento de las dos principales fórmulas de la contienda electoral.

3 La noción de prosumidor ha sido también utilizada para dar cuenta de las posibilidades que las nuevas tecnologías aportan no solo en el orden de la producción de información sino también de consumos culturales ya que se amplían las formas de creación y distribución en diversos campos como el audiovisual, musical, etc. (Fernández, 2014).

4 Este tipo de distinciones radicales también han sido identificadas entre los autores abocados al análisis de la globalización, fenómeno estrechamente vinculado con el desarrollo de las nuevas tecnologías de la información y la comunicación. En efecto, Perla Aronson (2007) señala que existen una serie de autores que observan dicho fenómeno con grandes expectativas y los caracteriza como hiperglobalizadores. Paralelamente, distingue otro grupo de autores que no consideran que la globalización constituya un fenómeno que obre en beneficio de aquellos sectores más desaventajados en el seno del capitalismo, a éstos últimos los caracteriza como escépticos.

5 En una nota publicada por La Nación pocos días antes del inicio de la temporada 2019 de su programa Periodismo Para Todos (PPT), emitido por Canal 13, Lanata afirma: «Ante todo, yo no inventé la grieta» (Stiletano, 24-05-2019).

6 Tal conflicto se desató durante el inicio del flamante gobierno de Cristina Fernández de Kirchner en el 2008 que lo enfrentó con los sectores agrarios

7 Para ilustrar brevemente esto bastan algunos ejemplos relacionados con los titulares del diario Clarín: Cristina aprovechó las grietas del G20 para criticar el ajuste fiscal, 26-06-2010; Las grietas en el modelo cristinista, 26-02-2012; Primeras grietas en el corazón del cristinismo, 20-03-2013.

8 Se trataría de aquella misma escisión que en contextos desarrollistas se planteó en términos del clivaje peronismoantiperonismo (Grimson, 2019, pp. 107-137; Ruiz del Ferrier y Tirenni, 2006, p. 144).

9 En efecto, la expresidenta Cristina Fernández de Kirchner afirmaba: «La grieta es una estrategia electoral del macrismo» (2019).

10 En este sentido, comprendían que la decisión de la exmandataria, intentaba suturar las diferencias con algunos dirigentes otrora muy cercanos y a los que podía volver a reunir en un frente electoral con posibilidad cierta de éxito en las urnas si renunciaba a encabezarlo. El 19 de mayo, tanto el diario Clarín como La Nación titulaban sendas notas a partir de la expresión del gobernador de La Pampa, Carlos Verna, quien se refería al carácter conciliador de la expresidenta. ("Carlos Verna: "Cristina busca cerrar la grieta"”, Clarín, 19.5.2019; “Carlos Verna: "Cristina Kirchner mostró amplitud e intenta cerrar la grieta"”, La Nación, 19.5.2019).

11 Título que, a su vez, repone el interrogante formulado por el histórico dirigente peronista Jorge Antonio en su libro publicado en 1966.

12 Conviene pues distinguir que, si bien se trata de un interés compartido por ciertos sectores del espectro político regional que resultan afines en su oposición a los gobiernos de corte populista, en cada caso nacional se presentan matices respecto del modo en que dichos sectores conjugaron sus posibilidades de acceder al poder. En 2015 en Argentina se dio por medio de elecciones libres, abiertas y competitivas. En 2018 en Brasil, país en el cual se encontraba bajo arresto el expresidente Luiz Inácio Lula da Silva, principal líder opositor, las condiciones fueron mucho más complejas y contradictorias. En ese país las elecciones se dieron luego del proceso de destitución presidencial de Dilma Rousseff llevado adelante en 2016 y formalmente denominado impeachment. Brasil resulta uno de los ejemplos más acabados, aunque no el primero ni el único de la estrategia articulada para clausurar alternativas progresistas sobre la base de la alianza entre el poder judicial y mediático (Lois y Wainer, 2019).

13 Según el Cambridge Dictionary el término rift se traduce como grieta, fisura. Dos ejemplos sobre este punto se pueden encontrar en los siguientes titulares: The Great Perceptual Rift (https://mitchellpsmith.com/2018/05/06/the-great-pe rceptual-rift/) y Make America whole again: how the US can heal its political rift (https://www.newscientist.com/articl e/mg23230981-600-make-america-whole-again-how-the-us-can-heal-its-political-rift/). 